\title{
THE VALUES OF A POLYNOMIAL OVER A FINITE FIELD
}

\author{
by S. D. COHEN
}

(Received 13 June, 1972)

1. The object of this paper is to derive, using a version of the large sieve for function fields due to J. Johnsen [6], explicit lower bounds for the average number of distinct values taken by a polynomial over a finite field.

Let $k$ be the finite field with $q$ elements, where $q$ is a positive power of some prime $p$. For a polynomial $f(x)$ in $k[x]$, define $V(f)$ to be the number of distinct values $f(\alpha)$ as $\alpha$ varies in $k$. For our purpose it is sufficient to consider only monic polynomials with zero constant coefficient. Therefore, take $f(x)$ to be the monic polynomial of degree $n$ given by

$$
f(x)=x^{n}+\sum_{i=1}^{n-1} \alpha_{i} x^{i} \quad\left(\alpha_{i} \in k\right)
$$

When $q$ is large, Birch and Swinnerton-Dyer [1, Theorem 2] have shown that $V(f)$ depends on a certain Galois group associated with $f$ in a manner made explicit by the author in $[4$, Theorem 2]. However, if some of the coefficients $\alpha_{i}$ are allowed to vary in $k$, then the author has also shown that, for large $q$, the average value of $V(f)$ depends only on $n$. Specifically, if the integer $t$ satisfies $0 \leqq t \leqq n-2$ and the $t$ coefficients $\alpha_{n-1}, \ldots, \alpha_{n-t}$ in (1) are given, define $v(n, t)\left(=v\left(n, t ; \alpha_{n-1}, \ldots, \alpha_{n-t}\right)\right)$ by

$$
v(n, t)=\sum_{a_{1}, \ldots, a_{n-t-1} \in k} V(f) / q^{n-t-1} .
$$

(Thus $v(n, t)$ is the average value of $V(f)$ over all monic polynomials (1) whose first $t+1$ coefficients are fixed.) Then Theorem 3 of [5] (see also (1.3) of [5]) implies that, if $p>n$ or, with a few exceptions, if $2<p \leqq n$, then, for fixed $n$,

$$
v(n, t)=\mu_{n} q+O\left(q^{1 / 2}\right),
$$

where

$$
\mu_{n}=1-(1 / 2 !)+\ldots+(-1)^{n-1} / n ! \text {. }
$$

Previously, S. Uchiyama [7] had shown that, if $p>n$, then $v(n, 0)$ is given explicitly by

$$
v(n, 0)=b(q, n) q,
$$

where $b(q, n)=\sum_{r=1}^{n}\left(\begin{array}{l}q \\ r\end{array}\right)(-1)^{r-1} q^{-r}$. In $\S 2$ below we provide a proof of (4) valid for all $n$ and $q$. Note that, if $\mu_{n}$ is given by (3), then, for fixed $n$, we have

$$
b(q, n)=\mu_{n}+O\left(q^{-1}\right) \quad(q \rightarrow \infty) .
$$

(In fact, Uchiyama [8] also proved that, for fixed $n<p$ and $t \geqq 1$,

$$
v(n, t)=b(q, n) \dot{q}+O\left(q^{t+1-(n / t)}\right) \quad(1 \leqq t \leqq n-1),
$$


an estimate which is nontrivial if $t^{2}<n$ and, in view of (5), better than (2) if $t\left(t+\frac{1}{2}\right)<n$.) Further, if $n \geqq q$, then obviously

$$
b(q, n)=1-\left(1-q^{-1}\right)^{q} \quad(n \geqq q) .
$$

It is evident that, as $n$ and $q$ both increase, $b(q, n)$ converges extremely rapidly to $1-e^{-1}=$ $0.632 \ldots$ Since $f(x) \equiv g(x)\left(\bmod x^{q}-x\right)$ implies $V(f)=V(g)$, it is not hard to see that, when $n-t \geqq q$, we can supplement (4) with

$$
v\left(n, t ; \alpha_{n-1}, \ldots, \alpha_{n-t}\right)=v(n, 0)=b(q, n) q \quad(n-t \geqq q),
$$

where, since $n \geqq q, b(q, n)$ is given by $(6)$.

In general, we therefore expect $v(n, t)$ to be approximately $b(q, n) q$. For large $q$, this is confirmed by (2) and (5). On the other hand, a lower bound for $v(n, t)$ close to this expected value for all $n$ and $q$ would seem to be of some interest. In this direction, L. Carlitz [3] proved that, if $p>n>1$, then $v(n, n-2) \geqq q^{2} /(2 q-1)>\frac{1}{2} q$, so that $v(n, t) \geqq q^{2} /(2 q-1)$ for all $t \leqq n-2$. Our purpose here is to prove the following theorem, which strengthens this result for $0 \leqq t<n-2$.

THEOREM 1. If $0 \leqq t \leqq n-2$ and $m=\left[\frac{1}{2}(n-t)\right]$ (in integral part notation), then

$$
v\left(n, t ; \alpha_{n-1}, \ldots, \alpha_{n-t}\right) \geqq c(q, m) q,
$$

where

$$
c(q, m)=1-\left\{\sum_{r=0}^{m}\left(\begin{array}{l}
q \\
r
\end{array}\right)(q-1)^{-r}\right\}^{-1} .
$$

Note that, since, for $m \geqq q$, we have $c(q, m)=1-\left(1-q^{-1}\right)^{q}$, then (7) implies that, for $n-t \geqq 2 q$, we actually have equality in (8). Further, for fixed $m$,

$$
c(q, m) \rightarrow 1-\{1+(1 / 2 !)+\ldots+(1 / m !)\}^{-1}, \text { as } q \rightarrow \infty .
$$

Hence, for increasing $m$ and $q, c(q, m)$ also converges rapidly to $1-e^{-1}$. When $t=n-2$, (8) is the inequality of Carlitz. For the next few even values of $n-t,(8)$ yields

$$
\begin{array}{ll}
v(n, n-4) \geqq 3 q^{2} /(5 q-2)>(3 / 5) q & (n \geqq 4), \\
v(n, n-6) \geqq q\left(10 q^{2}-11 q\right) /\left(16 q^{2}-23 q+6\right)>(5 / 8) q & (n \geqq 6, q \geqq 3), \\
v(n, n-8)>(41 / 65) q=(0.631 \ldots) q & (n \geqq 8, q \geqq 4) .
\end{array}
$$
$q^{d(A)}$.

In what follows we shall denote the degree of a polynomial $A$ by $d(A)$ and put $|A|=$

2. For completeness we include a proof of (4) valid for all $n$ and $q$. It is sufficient to evaluate $j(n)$, the number of monic polynomials of degree $n$ in $k[x]$ not divisible by a linear factor, because evidently $j(n)=q^{n}-v(n, 0) q^{n-1}$. For a full description of the simple zeta function technique that we employ, see [2]. 
For any non-zero $A$ in $k[x]$, let $\theta(A)=1$ if $A$ has no linear factor; otherwise let $\theta(A)=0$. If $s(>1)$ is real, then the zeta function

$$
\zeta(s)=\sum_{A}|A|^{-s}\left(=\sum_{n=0}^{\infty} q^{n(1-s)}\right)=\prod_{P}\left(1-|P|^{-s}\right)^{-1}
$$

(where the sum and product in (10) are over all monic $A$ in $k[x]$ and all monic irreducibles $P$ in $k[x]$, respectively) converges (to $\left.\left(1-q^{1-s}\right)^{-1}\right)$ and hence so does

It follows that

$$
\sum_{A} \theta(A) /|A|^{s}=\sum_{n=0}^{\infty} j(n) q^{-n s}=\prod_{\substack{P \\ d(P)>1}}\left(1-|P|^{-s}\right)^{-1} .
$$

$$
\sum_{n=0}^{\infty} j(n) q^{-n s}=\zeta(s) \prod_{\substack{P \\ d(P)=1}}\left(1-|P|^{-s}\right)=\zeta(s)\left(1-q^{-s}\right)^{q} \quad(s>1) .
$$

On equating coefficients of $q^{-n s}$ in (11), we obtain

from which (4) follows at once.

$$
j(n)=\sum_{r=0}^{n}(-1)^{r}\left(\begin{array}{l}
q \\
r
\end{array}\right) q^{n-r}
$$

3. We now cite a particular case of the large sieve inequality contained in the Corollary to Theorem 5 of [6]. Let $\mathscr{S}$ be a set consisting of $Z$ distinct polynomials of degree $\leqq N$ in $k[x]$, so that $Z \leqq q^{N+1}$. Let $W$ be a set of monic square-free polynomials of degree not exceeding $X=\left[\frac{1}{2}(N+1)\right]$ with the property that, to every monic irreducible $P$ dividing a member of $W$, there exists a set of $w(P)(>0)$ residue classes $(\bmod P$ ) such that all members of $\mathscr{S}$ belong to one of these residue classes $(\bmod P)$.

THEOREM 2 (Johnsen). Let $S=\sum_{F \in W} \prod_{P \mid F}(|P|-w(P)) / w(P)$, where the product is over all monic irreducibles dividing $F$. Then

$$
Z \leqq S^{-1} q^{N+1}
$$

4. Let $n$ be a given positive integer and $A, D, H$ be given polynomials in $k[x]$, with $d(H)<n$. Define $J(n, A, D, H)$ to be the number of polynomials $F$ with $d(F) \leqq n$ such that $F+A \equiv D(\bmod H)$ and such that $F+A$ has no linear factor in $k[x]$. We apply Theorem 2 to give an upper bound for $J(n, A, D, H)$ from which we deduce Theorem 1 .

THEOREM 3. Suppose that $d(H)=h$ and that $H$ has precisely $l$ distinct linear factors in $k[x]$. Then

where $M=\left[\frac{1}{2}(n-h+1)\right]$.

$$
J(n, A, D, H) \leqq\left\{\sum_{r=0}^{M}\left(\begin{array}{c}
q-l \\
r
\end{array}\right)(q-1)^{-r}\right\}^{-1} q^{-n-h+1},
$$

Proof. If $D_{1}$ is the unique polynomial such that $D_{1}=0$ or $d\left(D_{1}\right)<h$ and such that $D_{1} \equiv D-A(\bmod H)$, then clearly $J(n, A, D, H)=J\left(n, 0, D_{1}, H\right)$. Hence we may assume 
that $A=0$ and that $D=0$ or $d(D)<h$. Further, since the result is trivial if $D$ and $H$ have a common linear factor, we may also assume that this, in fact, is not the case. Let $k^{\prime}$ be the set of $q-l$ elements $\beta$ of $k$ such that $(x-\beta) \times H$. Then

$$
J(n, 0, D, H) \leqq J_{1}=\mid\left\{F \in k[x]: d(F) \leqq n, F \equiv D(\bmod H) \text { and } F(\beta) \neq 0 \forall \beta \in k^{\prime}\right\} \mid .
$$

Now to every $F$ counted in $J_{1}$, there exists a unique $G$ in $k[x]$ with $d(G) \leqq n-h$ such that $F=D+G H$. Hence

$$
\begin{aligned}
J_{1} & \leqq\left|\left\{G: d(G) \leqq n-h, D+G H \not \equiv 0(\bmod x-\beta) \forall \beta \in k^{\prime}\right\}\right| \\
& =\left|\left\{G: d(G) \leqq n-h, G(x) \not \equiv-(D(\beta) / H(\beta))(\bmod x-\beta) \forall \beta \in k^{\prime}\right\}\right| .
\end{aligned}
$$

We now apply Theorem 2 to the set of $G$ counted by (12). Put $N=n-h$ and let $W$ be the set of all square-free monic polynomials that are the products of not more than $\left[\frac{1}{2}(n-h+1)\right]=$ $M$ (distinct) linear factors prime to $H$, so that $w(x-\beta)=q-1$ for all $\beta \in k^{\prime}$. Obviously the number of polynomials in $W$ of degree $r(\leqq M)$ is $\left(\begin{array}{c}q-l \\ r\end{array}\right)$. Thus Theorem 2 yields $J_{1} \leqq$ $S^{-1} q^{n-h+1}$, where

$$
S=\sum_{F \in W(x-\beta) \mid F}(q-1)^{-1}=\sum_{r=0}^{M}\left(\begin{array}{c}
q-1 \\
r
\end{array}\right)(q-1)^{-r},
$$

and the theorem is proved.

Proof of Theorem 1. In the situation of Theorem 1, let $A(x)=x^{n}+\sum_{i=n-t}^{n-1} \alpha_{i} x^{i}$. Then clearly $v\left(n, t ; \alpha_{n-1}, \ldots, \alpha_{n-t}\right) q^{n-t-1}=q^{n-t}-J(n-t-1, A, 0,1) \geqq q^{n-t}\left\{1-\left[\sum_{r=0}^{m}\left(\begin{array}{l}q \\ r\end{array}\right)(q-1)^{-r}\right]^{-1}\right\}$

by Theorem 3, where $m=\left[\frac{1}{2}(n-t)\right]$, and the theorem follows.

We remark finally that, by using different choices of $A, D$ and $H$ in Theorem 3 , one could derive similar expressions for the average value of $V(f)$ over other sets of polynomials (e.g., those with the first $t+1$ and last $u$ (nonconstant) coefficients fixed).

\section{REFERENCES} 417-423.

1. B. J. Birch and H. P. F. Swinnerton-Dyer, Note on a problem of Chowla, Acta Arith. 5 (1959)

2. L. Carlitz, The arithmetic of polynomials in a Galois field, Amer. J. Math. 54 (1932), 39-50.

3. L. Carlitz, On the number of distinct values of a polynomial with coefficients in a finite field, Proc. Japan. Acad. 31 (1955), 119-120.

4. S. D. Cohen, The distribution of polynomials over finite fields, Acta Arith. 17 (1970), 255-271.

5. S. D. Cohen, Uniform distribution of polynomials over finite fields, J. London Math. Soc. (2) 6 (1972), 93-102.

6. J. Johnsen, On the large sieve method in GF [q, x], Mathematika 18 (1971), 172-184.

7. S. Uchiyama, Note on the mean value of $V(f)$, Proc. Japan. Acad. 31 (1955), 199-201.

8. S. Uchiyama, Note on the mean value of $V(f)$, II, Proc. Japan. Acad. 31 (1955), 321-323.

\section{UNIVERSITY OF GLASGOW}

GLASGOW G12 8QQ 